\title{
Hygro-Thermo-Mechanical Analysis and Failure Prediction in Electronic Packages by Using Peridynamics
}

\author{
Selda Oterkus and Erdogan Madenci \\ University of Arizona, Tucson, AZ \\ Erkan Oterkus \\ University of Strathclyde, Glasgow, UK \\ Yuchul Hwang, Jangyong Bae and Sungwon Han \\ Samsung Electronics Co., LTD, Korea.
}

\begin{abstract}
This study presents an integrated approach for the simulation of hygro-thermo-vapor-deformation analysis of electronic packages by using peridynamics. This theory is suitable for such analysis because of its mathematical structure. Its governing equation is an integro-differential equation and it is valid regardless of the existence of material and geometric discontinuities in the structure. It permits the specification of distinct properties of interfaces between dissimilar materials in the direct modeling of thermal and moisture diffusion, and deformation. Therefore, it enables progressive damage analysis in materials or layered material systems such as the electronic packages. It describes the validation procedure by considering a particular package for each thermomechanical, hygromechanical deformation as well as vapor pressure predictions. Also, it presents results concerning failure sites and mechanisms due to hygro-thermovapor-deformation.
\end{abstract}

\section{Introduction}

During packaging, transportation and storage, IC packages may absorb moisture leading to differential swelling between the polymeric and nonpolymeric materials, and among the polymeric materials. This differential swelling exacerbates the thermal deformation during the solder reflow process. In order to minimize the mismatch in swelling of the dissimilar materials of the IC packages during the solder reflow process, the packages are subjected to moisture conditioning (baking) for a period of time prior to this process. The estimate of baking period necessary to minimize the differential swelling and prevent possible cracking during the solder reflow process was investigated by Tay and Lin [1].

Although the baking process is essential in reducing the thermo-mechanical deformation during the solder reflow, it influences the moisture concentration distribution, and induces significant hygro-mechanical deformation. Also, the distribution of moisture concentration dictates the vapor pressure in micro voids while reducing the interfacial adhesion strength. The decrease in adhesion strength is caused by moisture absorption. Furthermore, the package cracking is not controlled by the absolute moisture content but its concentration at the critical interface, Kitano et al. [2]. An extensive discussion of moisture induced failure mechanisms in IC packages can be found in a study by Tee and $\mathrm{Ng}$ [3]
Coupled with the vapor pressure in micro voids, hygromechanical and thermo-mechanical deformation may cause interfacial delamination, and subsequent cracking at the padencapsulant interface, die-attach layer, and the die-encapsulant interface. Delamination and/or cracking at the die-attach layer is one of the primary failure mechanisms in plastic IC packages and often lowers the threshold for other mechanical, and electrical failures, Suhir [4] and Wong et al. [5].

The vapor pressure, dictated by the moisture concentration after baking, saturates much faster than the moisture diffusion, and that a near uniform vapor pressure is reached in the package, Tee and $\mathrm{Ng}$ [3]. The vapor pressure introduces an additional strain of the same order as that of the hygromechanical strains to the package. The hygro-mechanical stresses induced through moisture conditioning (baking) are significant compared to the thermo-mechanical stresses induced during the solder flow. Combination of these stresses can be detrimental to the reliability of the IC packages.

Therefore, the determination of the moisture concentration and temperature distributions is essential in order to determine the vapor pressure in micro voids, hygro-mechanical and thermo-mechanical stresses. There exists no known technique for the measurement of moisture distribution inside the package. Therefore, the predictive methods become unavoidable for investigating the effect of moisture conditioning.

Traditionally, moisture diffusion analysis is performed by using thermal-moisture analogy. Since the moisture concentration is not continuous along interfaces, a new parameter called "wetness" is introduced to render it continuous. Wetness is the ratio of the moisture concentration to its value at the saturated state, and it is continuous along interfaces. Although this approach is commonly accepted, it is not always valid because the saturated moisture concentration is not constant during the reflow process. A direct concentration approach (DCA) (Fan et al. [6]) should be employed to address this issue by imposing continuity condition along the interface between dissimilar materials.

A new continuum mechanics theory referred to as peridynamics (Silling [7]), removes this requirement because it is not necessary to impose continuity conditions. This feature of peridynamics emerges because the governing equations are based on integro-differential equations rather than partial differential equations of classical theory. 
Furthermore, peridynamics is also very suitable for failure prediction which allows cracks to initiate and grow naturally in the structure without resorting to any external crack growth law.

This study presents an integrated hygro-thermo-vapordeformation analysis using peridynamics to predict failure in electronic packages.

\section{Peridynamic (PD) Theory}

The peridynamic theory is a nonlocal continuum theory, and its continuum mechanics formulation was introduced by Silling [7] to overcome the difficulties arising due to the existence of discontinuities in the structure. The theory depends on integration rather than the spatial differentiation of PDEs as in classical continuum mechanics. Hence, it can be easily applicable to problems with discontinuities. As opposed to classical continuum mechanics, a material point inside the body can interact with other material points within its domain of influence called horizon, $\mathfrak{R}$ as shown in Fig. 1. The interaction (bond) between two material points $\mathbf{x}$ and $\mathbf{x}^{\prime}$ are expressed by using a response function, $\mathbf{f}$. Although PD formulation is originally given for mechanical field, it is applicable in other fields as well. The detailed derivation and capability of PD theory is given in the book by Madenci and Oterkus [8].

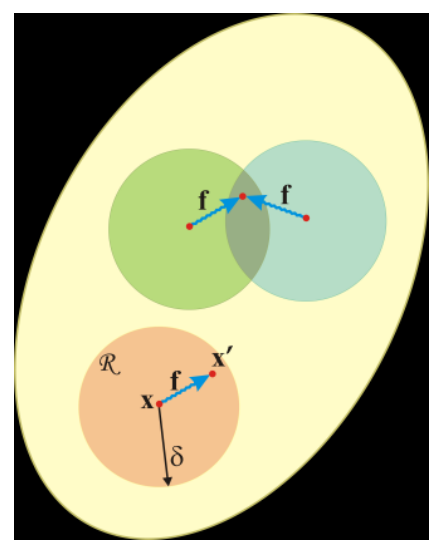

Fig. 1. Interaction of a material point with its neighboring points.

Basics - According to the PD theory, the field is analyzed by considering the interaction of a PD material point, $\mathbf{x}$, with the other, possibly infinitely many, material points in the body. Therefore, an infinite number of interactions may exist between the material point at location $\mathbf{x}$ and other material points. Hence, the PD state may contain particular information on an infinite number of interactions. However, the influence of the material points interacting with $\mathbf{x}$ is assumed to vanish beyond a local region (horizon), denoted by $\mathfrak{R}$ shown in Fig. 1. The range of material point, $\mathbf{x}$ is defined by $\delta$ referred to as the "horizon". Also, the material points within a distance $\delta$ of $\mathbf{x}$ is called the family of $\mathbf{x}, \mathfrak{R}$. The interaction of material points is prescribed through the response function which contains all of the constitutive information associated with the material. The response function also includes a length parameter (horizon), $\delta$. The locality of interactions depends on the horizon, and the interactions become more local with a decreasing horizon.

Hygrothermomechanics with vapor pressure - For known temperature and moisture concentration, the equation of motion of a material point can be expressed as

$\rho(\mathbf{x}) \ddot{\mathbf{u}} 、 \mathbf{x} t, \quad: \int_{\Re} \mathbf{f}\left(\mathbf{u}^{\prime}-\mathbf{u}, \mathbf{x}^{\prime}-\mathbf{x}\right) d V_{\mathbf{x}^{\prime}}+\mathbf{b}(\mathbf{x}, t)$

where the response function is defined as

$\mathbf{f}\left(\mathbf{u}^{\prime}-\mathbf{u}, \mathbf{x}^{\prime}-\mathbf{x}\right)=c\left(s-\alpha T_{\text {avg }}-\beta C_{\text {avg }}-\gamma p\right) \frac{\left(\mathbf{x}^{\prime}+\mathbf{u}^{\prime}\right)-(\mathbf{x}+\mathbf{u})}{\left|\left(\mathbf{x}^{\prime}+\mathbf{u}^{\prime}\right)-(\mathbf{x}+\mathbf{u})\right|}$

in which $c$ is the bond constant and can be expressed in terms Young's modulus, $E$

$c=\frac{9 E}{\pi h \delta^{3}}$ for $2 \mathrm{D}, c=\frac{12 E}{\pi \delta^{4}}$ for $3 \mathrm{D}$

with $h$ denoting the thickness.

The parameter $s$ represents the stretch between material points and given by

$s=\frac{\left|\left(\mathbf{x}^{\prime}+\mathbf{u}^{\prime}\right)-(\mathbf{x}+\mathbf{u})\right|-\left|\mathbf{x}^{\prime}-\mathbf{x}\right|}{\left|\mathbf{x}^{\prime}-\mathbf{x}\right|}$

where $\mathbf{u}$ and $\mathbf{u}^{\prime}$ are the displacements of material points, $\mathbf{x}$ and $\mathbf{x}^{\prime}$. Thermal related parameter $\alpha$ is the coefficient of thermal expansion and $T_{a v g}$ is defined as

$T_{\text {avg }}=\frac{\left(T-T_{0}\right)+\left(T^{\prime}-T_{0}\right)}{2}$

in which $T$ and $T^{\prime}$ are the temperature of material points $\mathbf{x}$ and $\mathbf{x}^{\prime}$ with $T_{0}$ being the reference temperature. Similarly, moisture related parameter $\beta$ is the coefficient of moisture expansion and $C_{\text {avg }}$ is defined as

$C_{\text {avg }}=\frac{\left(C-C_{0}\right)+\left(C^{\prime}-C_{0}\right)}{2}$

in which $C$ and $C^{\prime}$ are the moisture concentration of material points $\mathbf{x}$ and $\mathbf{x}^{\prime}$ with $C_{0}$ being the reference moisture concentration. Finally, vapor pressure related parameter $\gamma$ is the coefficient of vapor pressure expansion, i.e.

$\gamma=\frac{1-v}{E}$ for $2 \mathrm{D}, \gamma=\frac{1-2 v}{E}$ for $3 \mathrm{D}$

with $v$ being the Poisson's ratio, and $p_{\text {avg }}$ is defined as 
$p_{\text {avg }}=\frac{p+p^{\prime}}{2}$

in which $p$ and $p^{\prime}$ are the vapor pressure of material points $\mathbf{x}$ and $\mathbf{x}^{\prime}$.

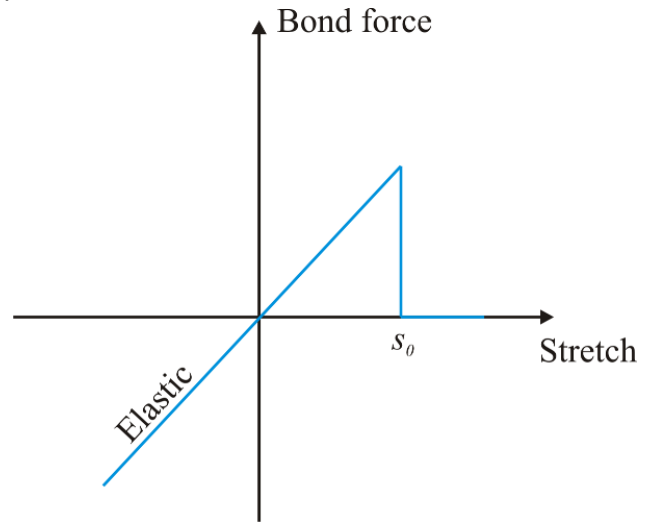

Fig. 2. Constitutive relation between material points in an elastic material.

In peridynamics, failure is introduced through a historydependent scalar-valued function, $\mu$, which is defined as

$\mu\left(t, \mathbf{x}^{\prime}-\mathbf{x}\right)=\left\{\begin{array}{cc}1 & \text { if } s\left(t^{\prime}, \mathbf{x}^{\prime}-\mathbf{x}\right)<s_{0} \text { for all } 0 \leq t^{\prime} \leq t \\ 0 & \text { otherwise }\end{array}\right.$

in which $s_{0}$ is the critical stretch where failure occurs, as shown in Fig. 2. Its value is calculated by the relationship

$s_{0}=\sqrt{4 \pi G_{f} /(9 E \delta)}$ for $2 \mathrm{D}, s_{0}=\sqrt{5 G_{f} /(6 E \delta)}$ for $3 \mathrm{D}$

where $G_{f}$ denotes the energy release rate. As suggested by Silling and Askari [9], an unambiguous notion of local damage at a point can be defined as

$\varphi(\mathbf{x}, t)=1-\left(\int_{\Re} d V_{\mathbf{x}^{\prime}} \mu\left(t, \mathbf{x}^{\prime}-\mathbf{x}\right) / \int_{\Re} d V_{\mathbf{x}^{\prime}}\right)$

Since the response function is nonlinear, the peridynamic equation of motion is solved numerically. Therefore, in order to carry out the numerical integration, the region of interest is first discretized into sub-domains in which the displacement and velocity fields are assumed to be constant. Hence, each sub-domain can be represented as a single collocation point located at the mass center of the sub-domain. After discretization, the peridynamic equation of motion can be cast as

$\rho^{. *} \square_{j} \because\left(\mathbf{u}, \mathbf{u}^{\prime}, \mathbf{x}, \mathbf{x}^{\prime}, t\right) V_{j}+\mathbf{b}\left(\mathbf{x}_{i}, t\right)$ in which $V_{j}$ is the volume of the sub-domain that is represented by the collocation point located at $\mathbf{x}_{j}$.

Thermal diffusion - As opposed to the deformation field, the peridynamic formulation for the thermal field concerns the interaction due to heat exchange between material points. The material points are connected through thermal bonds. The peridynamic heat conduction equation is derived as (Oterkus et al. [10])

$\rho c_{v^{i}}^{\dot{j}}, \quad, \quad \mathbf{J}_{\mathfrak{R}} f_{q}\left(T(\mathbf{x}, t), T^{\prime}\left(\mathbf{x}^{\prime}, t\right), \mathbf{x}^{\prime}, \mathbf{x}, t\right) d V_{\mathbf{x}^{\prime}}+q_{E}(\mathbf{x}, t)$

where $f_{q}$ is the thermal response function and $q_{E}$ is the joule heating term. The pairwise response function for isotropic materials can be written as

$f_{q}\left(\mathbf{x}^{\prime}, \mathbf{x}, t\right)=\kappa \frac{T\left(\mathbf{x}^{\prime}, t\right)-T(\mathbf{x}, t)}{\left|\mathbf{x}^{\prime}-\mathbf{x}\right|}$

in which the thermal bond constant, $\kappa$ is defined in terms of the thermal conductivity, $k$ as

$\kappa=\frac{6 k}{\pi h \delta^{3}}$ for $2 \mathrm{D}, \quad \kappa=\frac{6 k}{\pi \delta^{4}}$ for $3 \mathrm{D}$

Moisture diffusion - Direct approach for moisture diffusion analysis is essential (Fan et al. [6]). Similar to the heat conduction equation, the peridynamic formulation for the moisture concentration field concerns the interaction due to moisture exchange between material points. The material points are connected through hygro bonds. The peridynamic moisture concentration equation can be expressed as

$\left.i_{(\cdots,-,}\right]_{\mathfrak{R}} f_{c}\left(C(\mathbf{x}, t), C^{\prime}\left(\mathbf{x}^{\prime}, t\right), \mathbf{x}^{\prime}, \mathbf{x}, t\right) d V_{\mathbf{x}^{\prime}}$

where $f_{c}$ is the moisture concentration response function. The pairwise response function for isotropic materials can be written as

$f_{c}\left(\mathbf{x}^{\prime}, \mathbf{x}, t\right)=d \frac{C\left(\mathbf{x}^{\prime}, t\right)-C(\mathbf{x}, t)}{\left|\mathbf{x}^{\prime}-\mathbf{x}\right|}$

in which the hygro bond constant, $d$ is defined in terms of the moisture diffusivity, $D$ as

$d=\frac{6 D}{\pi h \delta^{3}}$ for $2 \mathrm{D}, d=\frac{6 D}{\pi \delta^{4}}$ for $3 \mathrm{D}$

Vapor pressure - Simulations of vapor pressure field are performed by adopting the procedure suggested by Fan et al. [11], in which they proposed a micro-mechanics approach. It is assumed that the voids in polymer materials are uniformly distributed, thus a representative volume element (RVE) can be used for a continuum representation of vapor pressure 
within the material. Fig. 3 shows a typical RVE with the moisture in both liquid and vapor state within the void. There exist three possibilities for the moisture state within the voids:

Case 1: The moisture within the void is in single vapor phase at pre-conditioning temperature $\left(T_{0}\right)$. Thus the moisture within the void is also in single vapor phase at reflow temperature $\left(T_{r}\right)$. In this case, the transition temperature $\left(T_{1}\right)$ at which all the moisture is transformed into vapor phase is less than the pre-conditioning temperature $\left(T_{1}<T_{0}\right)$.

Case 2: The moisture within the void is in mixed liquid and vapor phase at pre-conditioning temperature $\left(T_{0}\right)$ and in single vapor phase at reflow temperature $\left(T_{r}\right)$. In this case, the transition temperature $\left(T_{1}\right)$ is greater than the pre-conditioning temperature and less than the reflow temperature $\left(T_{0}<T_{1}<T_{r}\right)$.

Case 3: The moisture within the void is in mixed liquid and vapor phase at both pre-conditioning temperature $\left(T_{0}\right)$ and reflow temperature $\left(T_{r}\right)$. In this case, the transition temperature $\left(T_{1}\right)$ is greater than the reflow temperature $\left(T_{r}<T_{1}\right)$ thus the vapor pressure in the void is the saturated vapor pressure at reflow temperature $\left(p_{g}\left(T_{r}\right)\right)$ as given in steam tables.

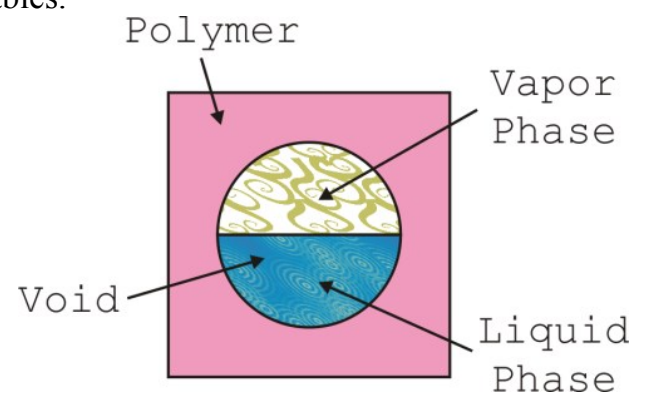

Fig. 3. Representative volume element (RVE) with a void.

Fig. 4 illustrates these three distinct cases. The vapor pressure, $p$ at each node is calculated by following the flowchart given in Fig. 5. Its saturated value is denoted by $p_{g}(T)$. In the computations, the following formulae are used for:

Case 1: $p=\frac{C \cdot p_{g}\left(T_{0}\right) \cdot T_{r}}{f \cdot \rho_{g}\left(T_{0}\right) \cdot T_{0}}$

Case $2: p=\frac{p_{g}\left(T_{1}\right) \cdot T_{r}}{T_{1}}$

Case 3: $p=p_{g}\left(T_{r}\right)$ in which, $f$ is the void volume fraction with its initial value being $f_{0}$. The moisture density and saturated moisture density are defined by $\rho_{m}=C / f_{0}$ and $\rho_{g}(T)$, respectively.

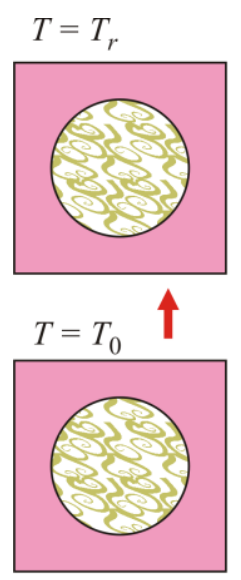

Case 1

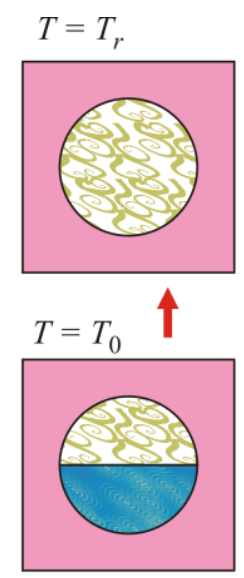

Case 2

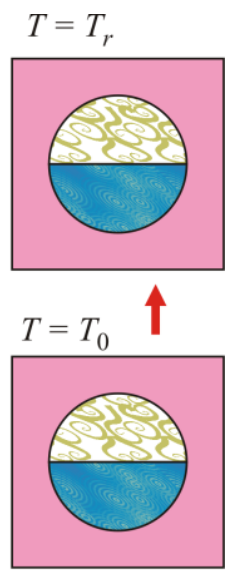

Case 3
Fig. 4. Three distinct moisture states in RVE.

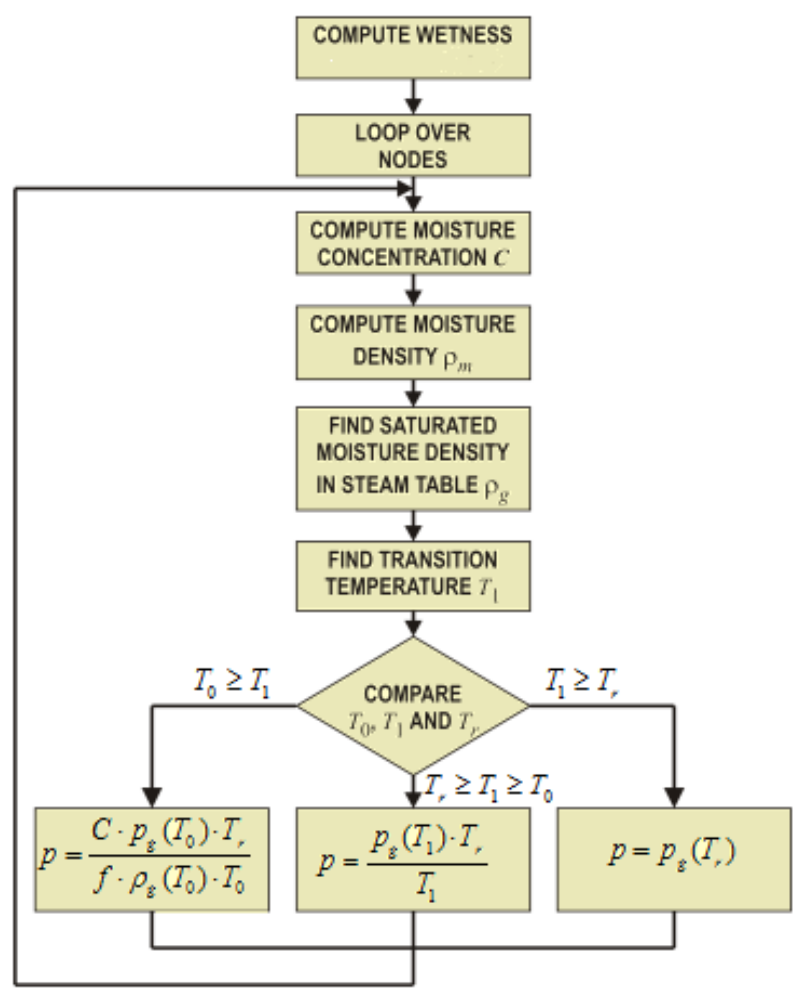

Fig. 5. Flowchart of vapor pressure simulations.

Saturated pressure $p_{g}(T)$ and saturated moisture density, $\rho_{g}(T)$ can be obtained from the steam tables. Their dependence on temperature is shown in Figs. 6 and 7. Furthermore, the variation of vapor pressure as a function of void volume fraction is depicted in Fig. 8. 


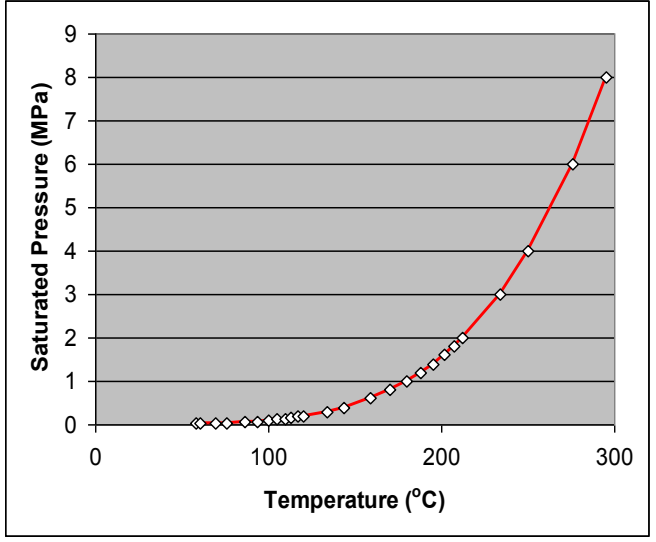

Fig. 6. Saturated pressure variation.

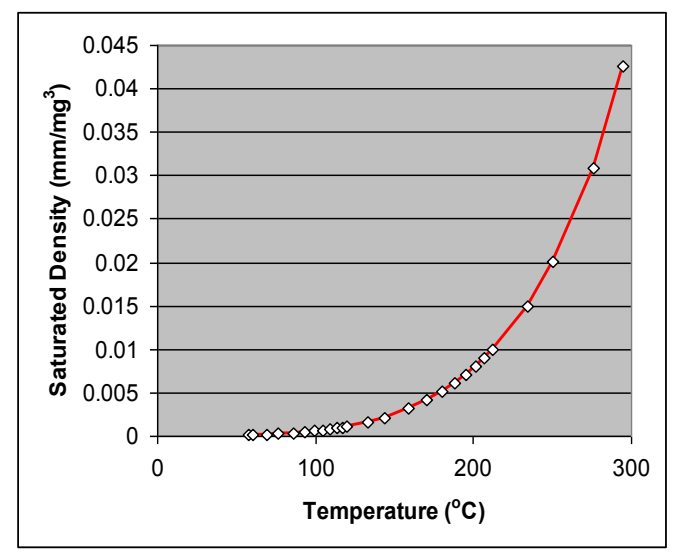

Fig. 7. Saturated moisture density variation.

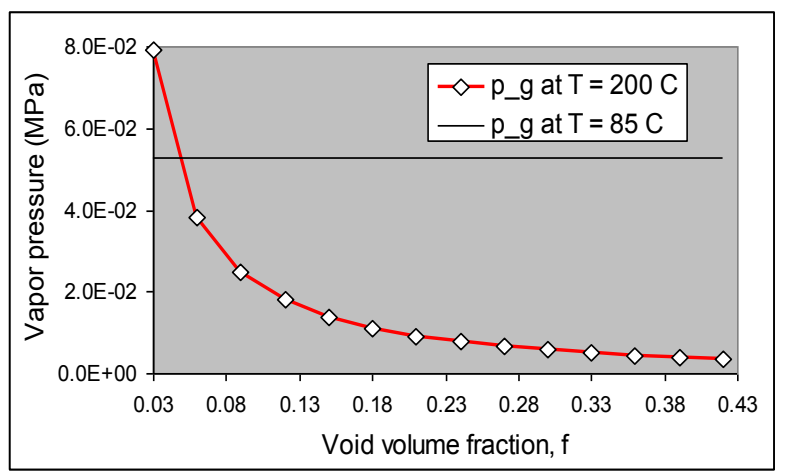

Fig. 8. Vapor pressure variation as a function of void volume fraction.

\section{Numerical results}

The validity of the peridynamic simulations is established by comparing the solutions for a particular package considered previously by Tee and $\mathrm{Ng}$ [3] against finite element predictions. The package geometry is described in Fig. 9.

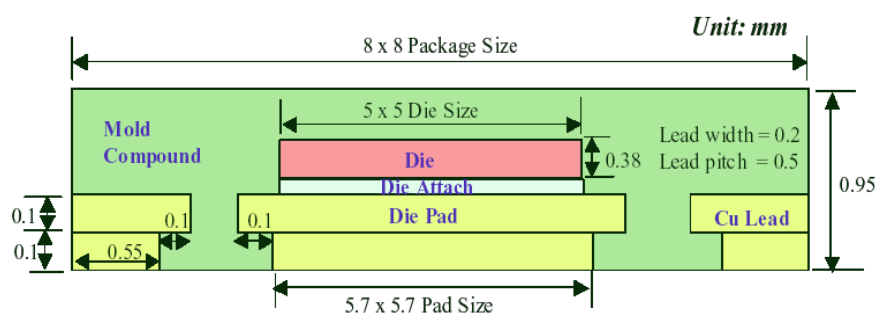

Fig. 9. Geometric and material configuration for the Quad Flat Non-Lead (QFN) package (figure taken from (Tee and $\mathrm{Ng}, 2000)$ ).

The geometric parameters are specified as shown in Fig. 10:

$$
\begin{aligned}
& L_{1}=2.5 \mathrm{~mm}, L_{2}=0.45 \mathrm{~mm}, L_{3}=0.4 \mathrm{~mm}, L_{4}=0.65 \mathrm{~mm} \\
& H_{1}=0.2 \mathrm{~mm}, H_{2}=0.05 \mathrm{~mm}, H_{3}=0.4 \mathrm{~mm}, H_{4}=0.35 \mathrm{~mm} \\
& h=0.01 \mathrm{~mm}
\end{aligned}
$$

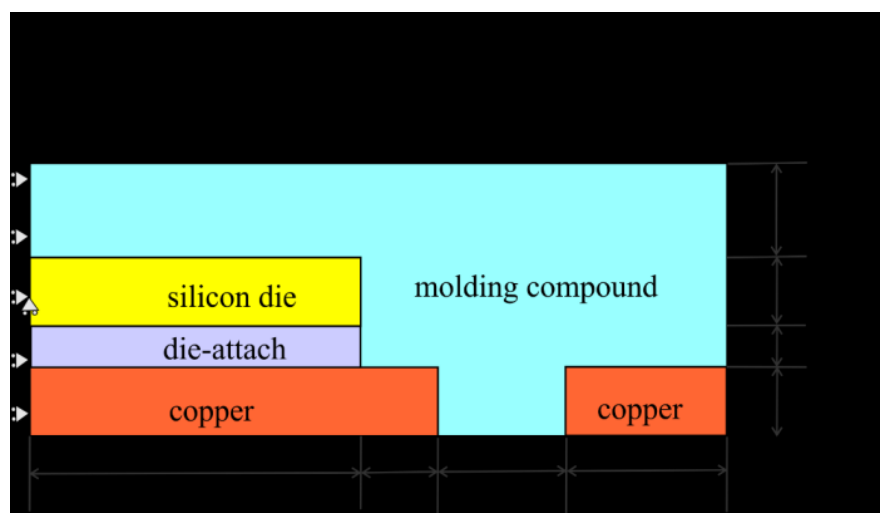

Fig. 10. Geometric parameters and symmetry conditions.

The material properties are specified as

$$
\begin{aligned}
& E_{1}=15 \times 10^{9} \mathrm{~Pa}, \quad E_{2}=7.4 \times 10^{9} \mathrm{~Pa}, \\
& E_{3}=163 \times 10^{9} \mathrm{~Pa}, \quad E_{4}=129 \times 10^{9} \mathrm{~Pa} \\
& v_{1}=0.25, \quad v_{2}=0.4, \quad v_{3}=0.278, \quad v_{4}=0.355 \\
& \beta C_{\text {sat } 1}=1.57 \times 10^{-3}, \quad \beta C_{\text {sat } 2}=3.22 \times 10^{-3}, \\
& \beta C_{\text {sat } 3}=0, \quad \beta C_{\text {sat } 4}=0 \\
& C_{\text {sat } 1}=7.06 \mathrm{~kg} / \mathrm{m}^{3}, C_{\text {sat } 2}=6.20 \mathrm{~kg} / \mathrm{m}^{3}, \\
& C_{\text {sat } 3}=0, \quad C_{\text {sat } 4}=0
\end{aligned}
$$

$$
\begin{aligned}
& \alpha_{1}=16 \times 10^{-6} 1 /^{o} \mathrm{~K}, \alpha_{2}=52 \times 10^{-6} 1 /^{o} \mathrm{~K}, \\
& \alpha_{3}=2.6 \times 10^{-6} 1 /^{o} \mathrm{~K}, \alpha_{4}=14.3 \times 10^{-6} 1 /^{o} \mathrm{~K}
\end{aligned}
$$

$$
\begin{aligned}
& \rho_{1}=1180 \mathrm{~kg} / \mathrm{m}^{3}, \rho_{2}=6450 \mathrm{~kg} / \mathrm{m}^{3}, \\
& \rho_{3}=2330 \mathrm{~kg} / \mathrm{m}^{3}, \rho_{2}=8940 \mathrm{~kg} / \mathrm{m}^{3}
\end{aligned}
$$


During absorption

$D_{1}=2.6748 \times 10^{-9} \mathrm{~m}^{2} / \mathrm{hr}$

During desorption

$D_{2}=45 \times 10^{-9} \mathrm{~m}^{2} / \mathrm{hr}$

$D_{1}=6.0 \times 10^{-7} \mathrm{~m}^{2} / \mathrm{hr}$

$D_{3}=1.0 \times 10^{-11} \mathrm{~m}^{2} / \mathrm{hr}$

$D_{2}=1.5 \times 10^{-6} \mathrm{~m}^{2} / \mathrm{hr}$

$D_{4}=1.0 \times 10^{-11} \mathrm{~m}^{2} / \mathrm{hr}$

$D_{3}=1.0 \times 10^{-11} \mathrm{~m}^{2} / \mathrm{hr}$

$D_{4}=1.0 \times 10^{-11} \mathrm{~m}^{2} / \mathrm{hr}$

in which subscript $1,2,3$ and 4 represent the molding compound, die attach, die and copper, respectively.

Thermomechanics - For thermomechanical deformation, the boundary conditions are specified as

$u_{x}(x=0, y, t)=0, u_{y}\left(x=0, y=H_{1}+H_{2}, t\right)=0$

and a uniform temperature change of

$\Delta T=1.0^{\circ} \mathrm{K}$

is assumed throughout the domain.

As shown in Figs. 11 and 12, the peridynamic and ANSYS predictions are in excellent agreement for thermomechanic deformations.
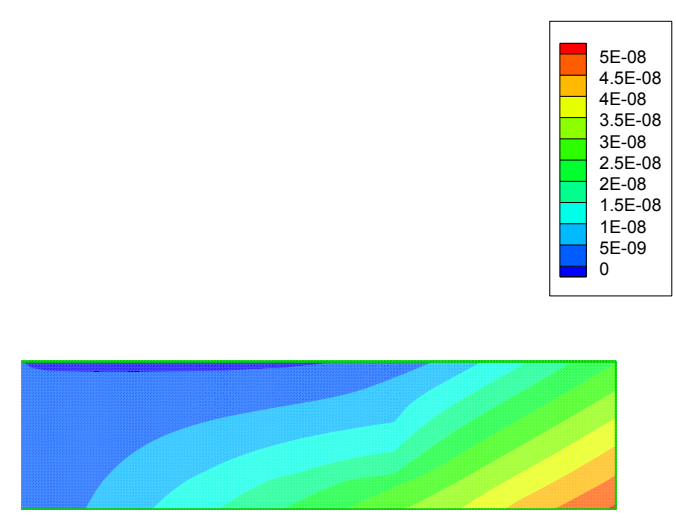

(a)
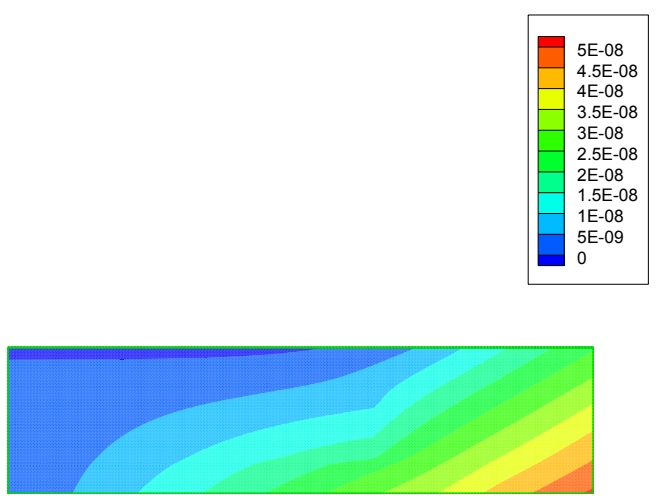

(b)

Fig. 11. $u_{x}$ variation in the package due to temperature change (a) PD results (b) ANSYS results

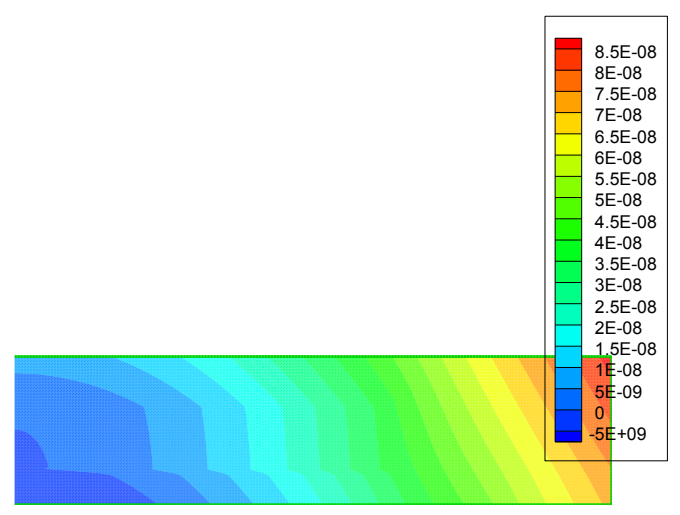

(a)

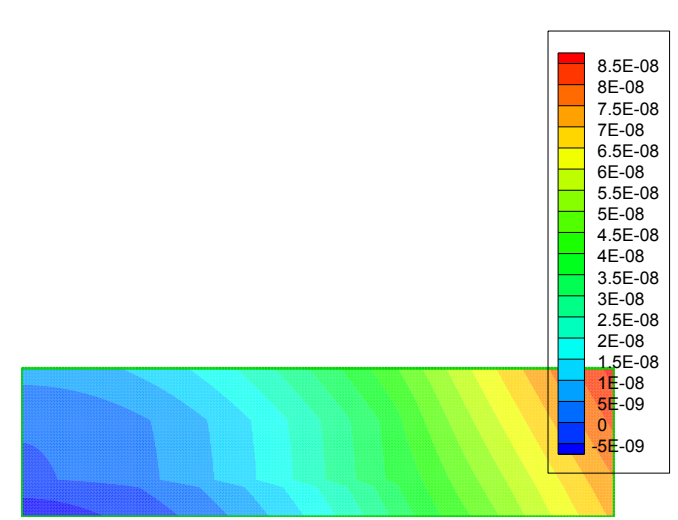

(b)

Fig. 12. $u_{y}$ variation in the package due to temperature change (a) PD results (b) ANSYS results

Hygromechanics - For hygromechanical deformation, the boundary conditions are specified as

$$
u_{x}(x=0, y, t)=0, \quad u_{y}\left(x=0, y=H_{1}+H_{2}, t\right)=0
$$

and the moisture concentration values in each material region are assumed as

$$
C_{1}=C_{\text {sat } 1}, C_{2}=C_{\text {sat } 2}, C_{3}=0, C_{4}=0
$$

As shown in Figs. 13 and 14, the peridynamic and ANSYS predictions are also in excellent agreement for hygromechanic deformations. 

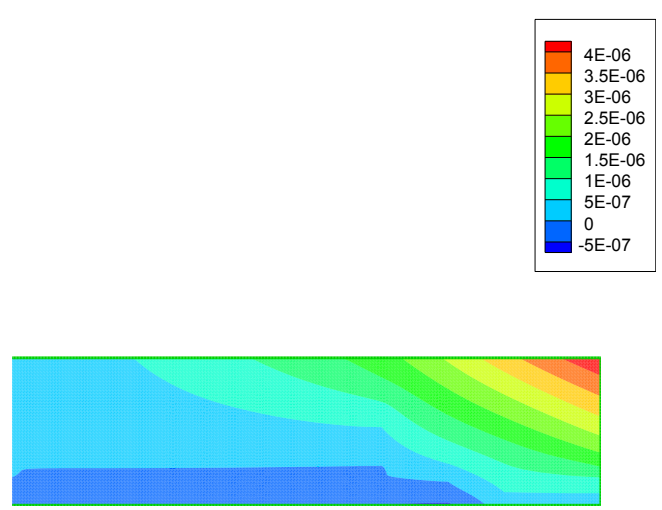

(a)
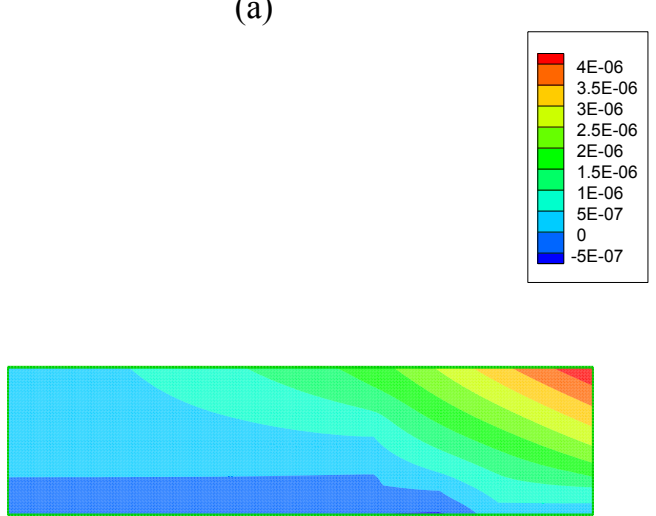

(b)

Fig. 13. $u_{x}$ variation in the package due to moisture concentration (a) PD results (b) ANSYS results

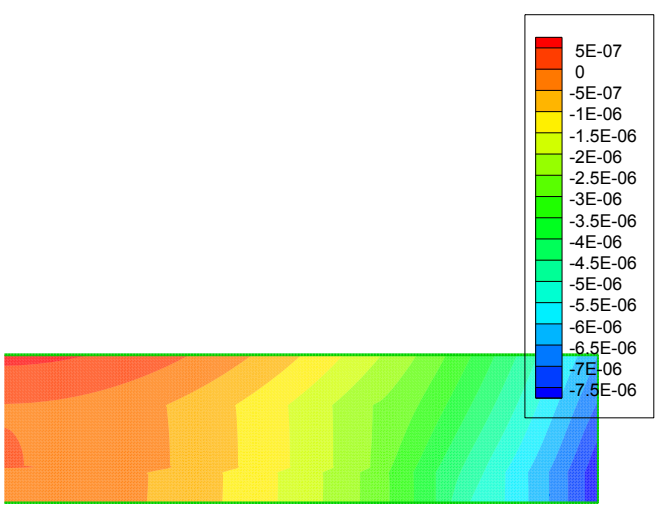

(a)

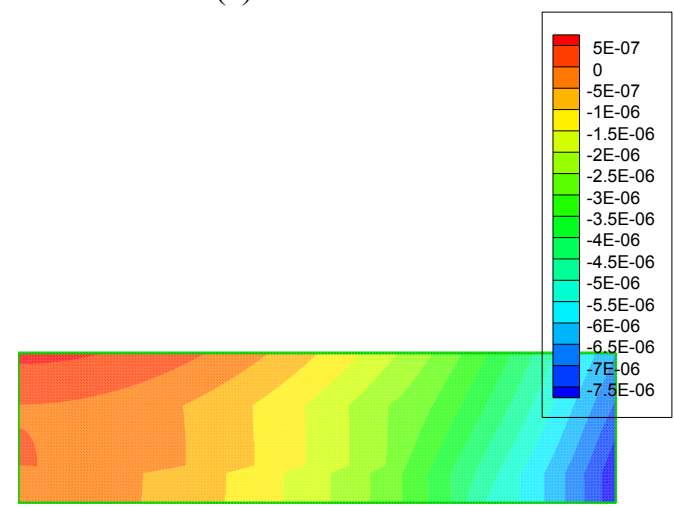

(b)

Fig. 14. $u_{y}$ variation in the package due to moisture concentration (a) PD results (b) ANSYS results
Vapor pressure distribution - In order to determine the effect of vapor pressure, first the package is subjected to $168 \mathrm{hr}$. absorption and $5 \mathrm{~min}$ desorption at $220^{\circ} \mathrm{C}$ (reflow temperature). The wetness distribution from peridynamic analysis is compared against ANSYS predictions as shown in Fig. 15 to validate the wetness results. Then, the desorption process is extended to $25 \mathrm{~min}$ and new wetness and corresponding concentration distributions are obtained as depicted in Fig. 16. At this concentration and temperature state, a maximum vapor pressure of $2.2 \mathrm{MPa}$ is obtained as demonstrated in Fig. 17. Corresponding deformations from both peridynamics and ANSYS predictions match very well as shown in Fig. 18 and 19.

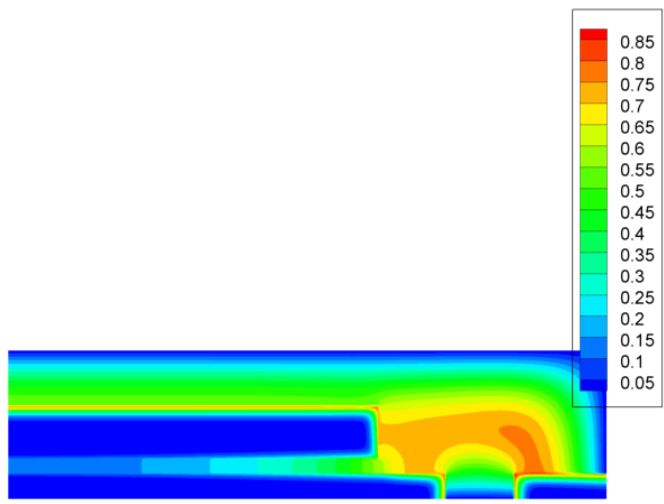

(a)

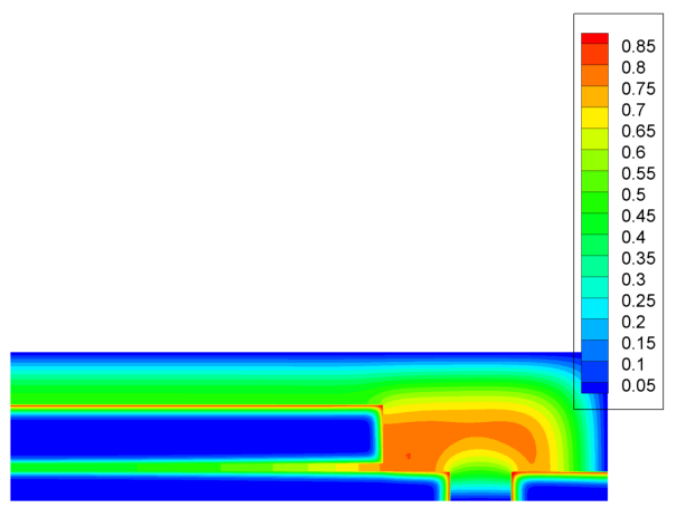

(b)

Fig. 15. Wetness distribution after absorption and desorption a) PD results b) ANSYS results (168hr. absorption and $5 \mathrm{~min}$ desorption) 


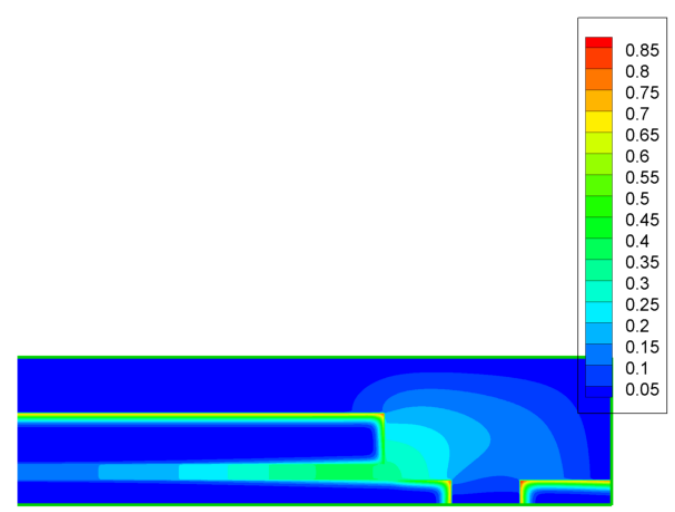

(a)

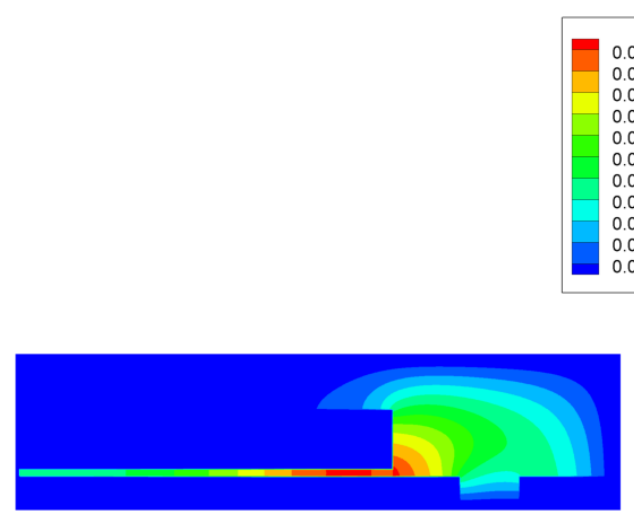

(b)

Fig. 16. PD results for a) wetness and b) corresponding concentration distribution after absorption and desorption (168hr. absorption and 25 min desorption)
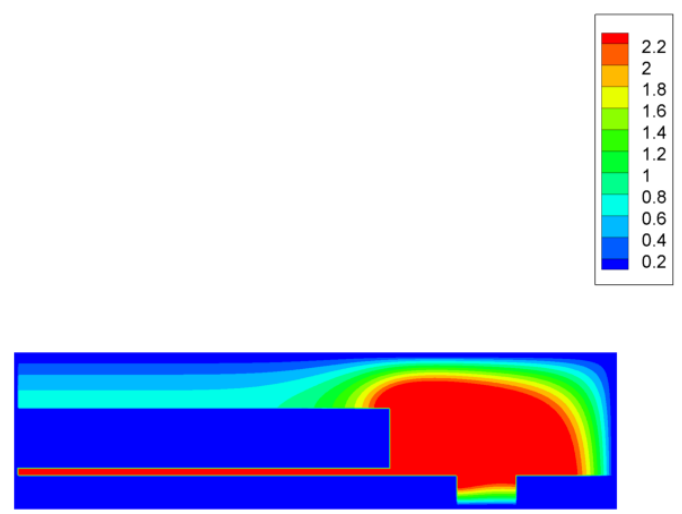

Fig. 17. Pressure distribution after absorption and desorption (MPa) (168hr. absorption and 25 min desorption)

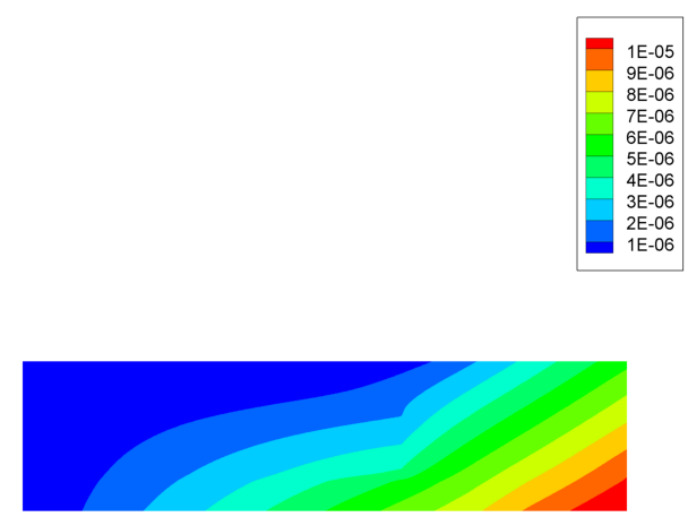

(a)

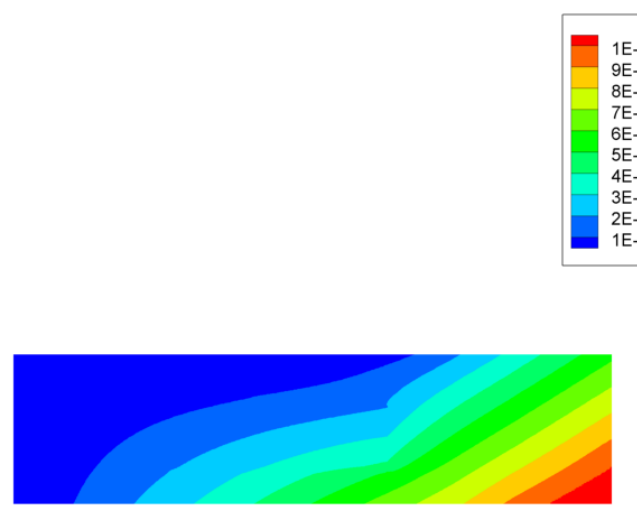

(b)

Fig. 18. $u_{x}$ variation in the package due to temperature change, concentration change and vapor pressure (a) PD results (b) ANSYS results 


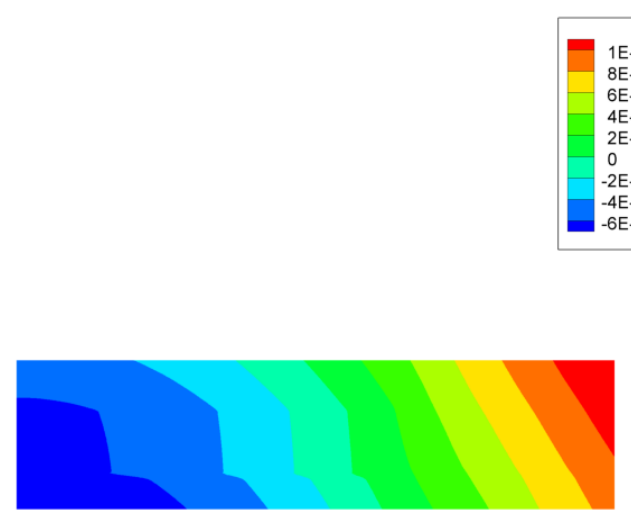

(a)

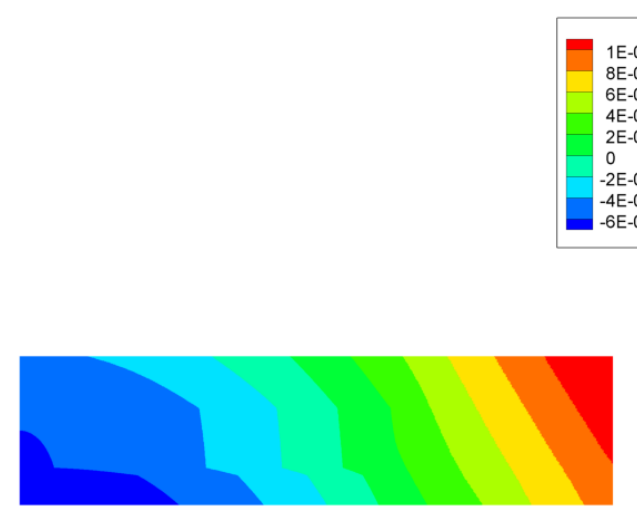

(b)

Fig. 19. $u_{y}$ variation in the package due to temperature change, concentration change and vapor pressure (a) PD results (b) ANSYS results

Failure prediction - In order to demonstrate the failure prediction capability of peridynamics, a critical stretch value of $s_{0}=0.015$ is assumed for all bonds inside the package. For this critical stretch value, it is observed that delamination/cracking occurs along the die-attach region and partial cracking occurs inside the molding compound as shown in Fig. 20. Extensive demonstration cases are left for a future study by considering different critical stretch values based on the individual material component and interface properties.

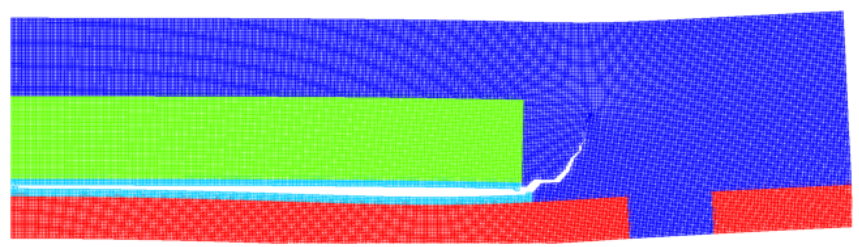

Fig. 20. PD failure prediction inside the package.

\section{Conclusions}

In this study, a new hygro-thermo-vapor-deformation analysis of electronic packages is presented by using peridynamics. As a result of the integro-differential formulation of peridynamics, it is straightforward to obtain moisture concentration without using "wetness" parameter by direct concentration approach (DCA). Furthermore, the formulation is also very suitable for failure analysis and for the demonstration case chosen, delamination/cracking failure along the die-attach region is observed which is a common failure mechanism in electronic packages.

\section{References}

1. Tay, A. A. O. and Lin, T. Y, 1998, "Quantifying the Effect of Prebaking on Delamination in Plastic IC Packages," EEPVol. 25, Workshop on Mechanical Reliability of Polymeric Materials and Plastic Packages of IC Devices, ASME, pp. 215-221.

2. Kitano, M., Nishimura, A., Kawai, S. and Nishi, K., 1988, "Analysis of Package Cracking During Reflow Soldering Process," IEEE/IRPS, pp. 90-95.

3. Tee, T. Y. and Ng, H. S., 2002, "Whole Field Vapor Pressure Modeling of QFN During Reflow with Coupled Hygro-mechanical and Thermo-mechanical Stresses," Proceedings, 52nd Electronic Components and Technology Conference, San Diego, California.

4. Suhir, E., 1997, " Failure Criterion for Moisture-Sensitive Plastic Packages of Integrated Circuit Devices: Application of Von-Karman's Equations with Consideration of Thermoelastic Strains," International Journal of Solids and Structures, Vol. 34, pp. 2991-3019.

5. Wong, E. H., Chan, K. C., Rajoo, R. and Lim, T. B., 2000, "The Mechanics and Impact of Hygroscopic Swelling of Polymeric Materials in Electronic Packaging," Proceedings, 50nd Electronic Components and Technology Conference, Las Vegas, Nevada.

6. Fan, X. J., Tee, T. Y., Shi, X. Q. and Xie, B., 2010, "Modeling of Moisture Diffusion and Whole-Field Vapor Pressure in Plastic Packages of IC Devices," in Moisture Sensitivity of Plastic Packages of IC Devices, X. J. Fan and E. Suhir (eds.), Springer, New York.

7. Silling S. A., 2000, "Reformulation of Elasticity Theory for Discontinuities and Long-range Forces," Journal of the Mechanics and Physics of Solids, Vol. 48, pp. 175-209.

8. Madenci, E. and Oterkus, E., 2013, Peridynamic Theory and Its Applications, Springer, New York.

9. Silling, S. A. and Askari, E., 2005, “A Meshfree Method Based on the Peridynamic Model of Solid Mechanics," Computers \& Structures, Vol. 83, pp. 1526-1535. 10. Oterkus, S., Madenci, E. and Agwai, A., 2014, "Peridynamic Thermal Diffusion, "Journal of Computational Physics, Vol. 265, pp.71-96. 
11. Fan, X. J., Zhang, G. Q. and Ernst, L. J., 2002, “A Micromechanics Approach for Polymeric Material Failures in Microelectronic Devices," Proceedings of the conference EuroSimE 2002, Paris, France, pp. 154-164. 